\title{
From standardization to personalization in breast cancer treatment
}

\author{
Shinzaburo Noguchi · Masakazu Toi
}

Published online: 7 April 2009

(c) The Japanese Breast Cancer Society 2009

One of the most important goals of cancer treatment, including that of breast cancer, is the realization of personalized medicine, and for achieving this goal, it is indispensable to develop new diagnostic methods for predicting patient prognosis with a higher accuracy than conventional imaging modalities and pathological diagnosis as well as for predicting the response to chemotherapy, hormonal therapy or molecular targeted therapy with greater precision. New methods for predicting the risk of developing breast cancer are also very important when personalization of breast cancer screening and indications of chemoprevention are considered, especially in Japanese women who have an apparently lower breast cancer incidence (one-third) than Caucasians.

In the symposium entitled "From standardization to personalization in breast cancer treatment," six outstanding speakers were invited to talk about their recent research on the development of new diagnostic methods in consideration of future application to personalized medicine. Excerpts were as follows.

The first speaker was Dr. Yasuhiro Tamaki from the Department of Breast and Endocrine Surgery, Osaka University Graduate School of Medicine, Osaka, Japan, who presented their recent results on the development of new risk

\footnotetext{
S. Noguchi $(\square)$

Department of Breast and Endocrine Surgery,

Osaka University Graduate School of Medicine,

2-2 Yamadaoka, Suita, Osaka 565-0871, Japan

e-mail: noguchi@onsurg.med.osaka-u.ac.jp

M. Toi

Department of Breast Surgery,

Kyoto University Graduate School of Medicine,

Kyoto, Japan

e-mail: toi@kuhp.kyoto-u.ac.jp
}

factors for breast cancer with a special reference to those related to obesity as well as mammographic breast density determined by their newly developed software. They clearly showed that obesity is associated with postmenopausal breast cancer risk through the elevation of serum estrogen levels and down-regulation of adiponectin levels. Since normal breast epithelial cells express adiponectin receptors type 1 and 2 , but no adiponectin, it is speculated that adiponectin produced in the adipose tissue down-regulates the normal breast epithelial cell growth in an endocrine manner. They have also succeeded in the development of a software that can measure the mammographic breast density and have shown that high breast density is associated with breast cancer risk. These risk factors seem to have a potential to be used as tools to estimate the breast cancer risk and thus personalize breast cancer screening.

The second speaker was Dr. Kikuya Kato from the Research Institute, Osaka Medical Center for Cancer and Cardiovascular Diseases, Osaka, Japan. He comprehensively reviewed the recent development of gene expression, profiling technologies and their application to the diagnosis of patient prognosis and responsiveness to chemotherapy/ hormonal therapy. His talk was very educational for the audience, which was mostly composed of surgeons who were not familiar with this research field. A better understanding of these new technologies is becoming increasingly important each year since molecular-based diagnostic methods, such as Oncotype DX and MammaPrint, have recently been introduced into practice, and new methods are expected to be introduced soon. In such circumstances, we surgeons are required to understand the results of these new diagnostic methods correctly and to utilize them in our practice for better treatment of patients.

The third speaker was Dr. Yoshio Miki from the Department of Molecular Diagnosis, the Cancer Institute, 
Japanese Foundation for Cancer Research, Tokyo, Japan. $\mathrm{He}$ is one of the top researchers in the field of gene expression analysis in human breast cancer and its clinical application. He mostly talked about the recent results obtained by his research group using DNA microarray analysis of breast cancer cells microdissected from breast cancer tissues. They have succeeded in developing a new diagnostic system using DNA microarray for the prediction of response to chemotherapy (paclitaxel, etc.) and are now conducting an innovative clinical trial to prove the value of their diagnostic system. Their results seem to be very promising, and the results of the clinical trial are eagerly awaited.

The fourth speaker was Dr. Shinichiro Horiguchi from the Department of Pathology, Tokyo Metropolitan Komagome Hospital. He is an expert in molecular pathological diagnosis. Based upon the clinic-pathological outcomes of the Japan Breast Cancer Research Group (JBCRG) 01 trial, he focused on the clinical implication of measurement of topoisomerase II as well as HER2 by FISH for predicting pathological response to preoperative multidrug chemotherapy consisting of an anthracycline-containing regimen and taxane and postoperative survival. It seems that pre-treatment topoisomerase II overexpression correlates with the pathological response, and posttreatment topoisomerase II and HER2 overexpression associates with poor survival. The clinical usefulness and controversial issues were discussed.

The fifth speaker was Dr. Sadako Akashi from the Department of Surgery, National Cancer Center Hospital, Tokyo. She is an expert on surgical treatment and is also interested in neoadjuvant treatments for primary breast cancer. She talked of the importance of imaging diagnosis for the individualization of local treatment, especially surgery. Results of Dr. Akiyama's research project, a MHLW cancer grant, were presented to discuss the role of
CT and of other diagnostic modalities such as MRI in assessing tumor extension and the level of surgical removal. The issue of axillary diagnosis was also mentioned; minimal and precise resection of tumor depends upon sophisticated imaging diagnosis for each person. This is true, but not easy in practice, so that real experience and several suggestions for improvements were shared.

The sixth and last speaker was Dr. Shozo Ohsumi from the Department of Surgery, Shikoku Cancer Center of the Hospital Matsuyama. He presented the outcomes of a nonirradiation study from the Dr. Inaji's research project for the MHLW cancer grant. It was reported that 123 primary breast cancer patients having low risk factors for local recurrence, such as less than $3 \mathrm{~cm}$ tumor size, node negative, over 50 years old, estrogen receptor positive and $5 \mathrm{~mm}$ tumor free margin, were enrolled in this study. All patients received breast-conserving surgery. Neither radiation therapy nor chemotherapy was conducted, but hormonal treatment with tamoxifen and/or anastrozole was performed after surgery. At the 36-month mean follow-up, six events, including three local relapses, one contralateral breast cancer, one distant metastasis and one uterus cancer occurrence, were observed. It was suggested that the issue of radiotherapy indications should be considered for patients having low risk of local recurrence after breastconserving surgery.

Recent developments in genome science have culminated in the development of Oncotype DX and MammaPrint in the field of breast cancer. These new diagnostic methods are the first molecular-based methods that are successfully translated into daily practice for breast cancer in the prediction of patient prognosis with a higher accuracy than the conventional histological diagnosis. This epoch-making event seems to have opened the door for a new era when "from standardization to personalization" is realized in breast cancer treatment. 